\title{
Absence of significant endocrine deficiencies in benign intracranial hypertension
}

\author{
ALISON C REIDAND JOH N A TOMSON \\ From the Glasgow University Departments of Neurology and Medicine, Glasgow, Scotland
}

SUMMARY Hypothalamic-pituitary function tests have been carried out in young, obese females with both recently diagnosed and longstanding benign intracranial hypertension. No abnormalities have been found which could be implicated in the pathogenesis of the condition, nor have we been able to demonstrate any convincing evidence of long-term hypothalamic pituitary damage. The results of these investigations can be difficult to interpret and invalid conclusions may be drawn if all factors involved are not carefully considered.

The syndrome of benign intracranial hypertension is thought to be due to brain swelling, ${ }^{1}$ but the underlying pathogenic mechanisms are still poorly understood. An endocrine disturbance has been inferred since the condition is found typically in young obese women, of ten with menstrual irregularities or taking an oral contraceptive pill, in patients with Addison's disease,${ }^{23}$ during reduction of corticosteroid therapy, ${ }^{45}$ and in treatment of hyperadrenal states. ${ }^{6}$ In a recent report it has been claimed that hypothalamic-hypophyseal insufficiency may result from the condition. ${ }^{7}$ Tests of anterior pituitary function have been performed on patients with the disease for both short and prolonged periods, to determine their value in elucidating any underlying disturbance or as prognostic aids.

\section{Patients and methods}

\section{Patients with "acute" and "chronic" benign} intracranial hypertension

Five obese female patients with active benign intracranial hypertension which had been diagnosed within the last three months were studied. In three patients the test was conducted prior to commencement of treatment, and the other two were being treated with acetazolamide and lumbar punctures. Tests were also carried out in another five obese female patients in

Address for reprint requests: Dr Alison C Reid, Glasgow University Department of Neurology, Institute of Neurological Sciences, Southern General Hospital, Glasgow G51 4TF.

Accepted 7 April 1981 whom the length of time since diagnosis ranged from 14 months to 13 years (mean 7 years 2 months). At the time of the study the disease was still active as the patients had persisting papilloedema and raised intracranial pressure. Current treatment was analgesics alone (two) and acetazolamide (two). One patient had a non-functioning ventriculoperitoneal shunt. None of the patients had received corticosteroids in the preceding two years.

\section{Control subjects}

There were 10 similarly age-matched obese young women who had been investigated at the Royal Infirmary, Glasgow, for amenorrhoea or for suspected Cushing's syndrome. In all patients a diagnosis of simple dietary obesity had been made after exclusion of any endocrine disease.

\section{Methods}

Anterior pituitary function was assessed in response to insulin-induced hypoglycaemia $(0.2$ units soluble insulin per $\mathrm{kg})$, thyrotropin releasing hormone $(200 \mu \mathrm{g})$ and gonadotropin releasing hormone $(100 \mu \mathrm{g})$ after an overnight fast. In each patient symptomatic hypoglycaemia was produced and the blood sugar level fell well below $2.0 \mathrm{mmol} / 1$. Blood samples were taken for blood sugar, cortisol, growth hormone, TSH, FSH, LH and prolactin levels at $0,30,60,90$, and 120 minutes from an indwelling venous catheter. Hormone assays were performed in the Radioimmunoassay Department of the Royal Infirmary and the Biochemistry Department of the Southern General Hospital, Glasgow. 


\section{Results}

The basal and peak responses of each hormone is shown in the table. Basal cortisol levels showed that the patients were not unduly stressed prior to commencement of the test and a normal rise in cortisol level occurred in all cases. A normal peak growth hormone response occurred in four patients with "acute" benign intracranial hypertension and in one, a borderline response of 15 $\mathrm{mU} / 1$ was obtained. Three of the five patients with "chronic" benign intracranial hypertension and one obese control had subnormal responses. Total $\mathrm{T}_{4}$ and $\mathrm{T}_{3}$ levels were normal in all patients. The increment in TSH in response to TRH was normal apart from one obese control in whom a marginally high response of $26.6 \mathrm{mU} / 1$ was obtained.

A rise in FSH occurred in all cases but the responses were unusually brisk, with high levels of $37 \mathrm{U} / 1$ and $29.9 \mathrm{U} / 1$ in two patients with "acute" benign intracranial hypertension. The response of LH was also very brisk for young premenopausal women in several cases in all three groups. Prolactin levels were not measured in all the control subjects. A normal rise in prolactin was obtained in all patients with "acute" benign intracranial hypertension. A normal response was also seen in four patients in the "chronic" group, but one had a subnormal response.

\section{Discussion}

Abnormalities found in this type of study must be interpreted in the light of the following questions. Are they an indication of an underlying primary endocrine disturbance, a reversible consequence of raised intracranial pressure, a manifestation of permanent damage to hypothalamic-pituitary structures, merely a feature of obesity, artifactual due to previous treatment with corticosteroids, or a function of a poorly performed test? If all these variables are not carefully considered, invalid conclusions may be drawn.

In this study the control subjects were similar in that they were age and sex matched and also obese. It could be argued that control subjects for a study such as this should also have raised intracranial pressure in order to determine if this alone may produce reversible secondary disturbances of hypothalamic-pituitary function. We did not consider it ethical however, to submit ill patients, for example those with raised intracranial pressure due to brain tumours, to this type of study for this purpose alone.

Oldstone $^{8}$ found an abnormal response to metyrapone in patients with acute benign intracranial hypertension which reverted to normal after the conditions had resolved. He merely assumed, however, that the adrenal gland had been effectively blocked after administration of metyrapone, and presented no data to confirm this in the form of subnormal serum or urinary cortisol levels. Oldstone concluded that his findings could be either a reversible secondary effect of the raised intracranial pressure or may represent a primary initiating cause of the syndrome. Nevertheless all the patients with acute benign intracranial hypertension in this study had normal cortisol responses.

The interpretation of the abnormal growth hormone response in three of the five patients with "chronic" active benign intracranial hypertension is difficult. It could represent damage to hypothalamic-pituitary structures but it could also be merely a feature of obesity. It has been reported that in patients with raised intracranial pressure due to hydrocephalus, permanent damage to the hypothalamic-pituitary region may be sustained. ${ }^{9}$ On the other hand cortisol, growth hormone and prolactin responses may all be impaired in persons with gross obesity alone. ${ }^{10}$ It is of interest to note that one patient with "acute" benign intracranial hypertension had a borderline growth hormone response and one control subject had an abnormal response, suggesting that obesity may have been the important common factor.

The care with which tests are performed is another important factor for consideration. Barber and Garvan ${ }^{7}$ claimed that five out of their eight patients with benign intracranial hypertension had inadequate cortisol responses to insulin-induced hypo glycaemia. Examination of the data, however, showed that the "basal" cortisol and growth hormone levels were high, suggesting that their patients were stressed prior to commencement of the test. It is also invalid to perform these tests on patients who have been treated with corticosteroids in the preceding months as a degree of adrenal suppression may still be present.

The very brisk FSH and LH responses in several patients in all three groups are interesting and the interpretation must be speculative. A possible explanation could be that abnormal peripheral steroid interconversions in extraglandular fat tissue $^{11}$ may have led to abnormal feedback of sex hormones at the hypothalamic level.

This study has not revealed any consistent abnormalities with conventional tests of anterior pituitary function which could be implicated in the pathogenesis of the condition. Furthermore, it has not shown any convincing evidence of long- 





term hypothalamic-pituitary damage resulting from the condition. Patients with benign intracranial hypertension must be actively treated because of the dangers of visual impairment, not because of endocrinological considerations.

\section{References}

1 Reid AC, Matheson MS, Teasdale G. Volume of the ventricles in benign intracranial hypertension. Lancet 1980; 2:7-8.

2 Jefferson A. Clinical correlation between encephalopathy and papilloedema in Addison's disease. J Neurol Neurosurg Psychiatry 1956; 19: 21-7.

3 Walsh FB. Papilloedema associated with increased intracranial pressure in Addison's disease. Arch Ophthalmol 1952; 47:86.

4 Greer M. Benign intracranial hypertension II. Following corticosteroid therapy. Neurology (Minneap) 1963; 13:439-41.

5 Neville BGR, Wilson J. Benign intracranial hypertension following corticosteroid withdrawal in childhood. Br Med J 1970; 3:554.

6 Newman PK, Snow M, Hudgson P. Benign intracranial hypertension and Cushings disease. $B_{r}$ Med J 1980; 2:113.

7 Barber SG, Garvan N. Is "benign intracranial hypertension" really benign? J Neurol Neurosurg Psychiatry 1980; 43:136-8.

8 Oldstone MA. Disturbance of pituitary adrenal relationships in benign intracranial hypertension (pseudotumor cerebri). J Clin Endocrinol 1966; 26:1366-9.

9 Barber SG, Garvan N. Hypothalamic hypopituitarism in normal pressure hydrocephalus. Lancet 1978; 2:1079.

10 Kopelman PG, Pilkington TRE, Jeffcoate SL, White N. Persistence of defective control of prolactin secretion in some obese women after weight reduction. $\mathrm{Br}$ Med $J$ 1980; 2:358-9.

11 Siiteri PK, MacDonald PC. Role of extraglandular oestrogen in human endocrinology. In: Green RO ed. Handbook of Physiology, Section 7, Endocrinology, Vol 11, Part 1, 615-29. Baltimore: Williams and Williams. 